\title{
A Re-Evaluation of the Effect of Trauma Center Verification Level on the Early Risk of Death in Hemodynamically Unstable Patients
}

\author{
David Plurad ${ }^{1}$, Glenn Geesman ${ }^{2}$, Nicholas Sheets ${ }^{1}$, Bhani Chawla-Kondal ${ }^{1}$, Ahmed Mahmoud ${ }^{2}$ \\ 1. Trauma and Acute Care Surgery, Riverside Community Hospital, Riverside, USA 2. General Surgery, Riverside \\ Community Hospital, Riverside, USA
}

Corresponding author: David Plurad, david.plurad@envisionhealth.com

\section{Abstract \\ Background}

Studies show increased early and overall mortality at level II compared to level I trauma centers in hemodynamically unstable patients. We hypothesize there is no mortality difference between level I and level II centers applying more contemporary data.

\section{Study design}

Utilizing the 2017 Trauma Quality Program Participant Use File (TQP-PUF), we identified adult patients (age $>14$ years) who presented to an American College of Surgeons (ACS) verified level I or II center with hypotension (systolic blood pressure $[\mathrm{SBP}]<90 \mathrm{mmHg}$ ). Logistic regression was performed to identify adjusted associations with mortality.

\section{Results}

A total of 7,264 patients met the inclusion criteria, of whom most were males $(4,924$ [67.8\%]) with blunt trauma (5,924 [81.6\%]) being predominated. Mean admission SBP was $73.2( \pm 13.0) \mathrm{mmHg}$. There were 1,097 (15.1\%) deaths. Level I admissions (4,931 (67.9\%]) were more likely male (3,389 [68.7\%] vs. 1,535 [65.8]; $\mathrm{p}=0.012)$, non-white $(3,119$ [63.3\%] vs. 1,664 [71.3\%]; $\mathrm{p}<0.001)$, a victim of penetrating trauma (933 [18.9\%] vs. 385 [16.5\%]; p=0.015), and more severely injured (mean Injury Severity Score: $19.3\left[{ }^{ \pm 15}\right]$ vs. $16.7[ \pm 13.7]$; $\mathrm{p}<0.001)$. Level II admissions (2,333 [32.1\%]) were older $(46.8[ \pm 18.5]$ vs. $50.3[ \pm 20.1]$ years; $\mathrm{p}<0.001)$ with more co-morbidities (mean Charlson Comorbidity Index: 1.43 [ \pm 2$]$ vs. 1.77 [ \pm 2.2$] ; \mathrm{p}<0.001$ ). Adjusted mortality between level I and II admissions was similar (766 [15.5\%] vs. 331 [14.2\%]; p=0.918). Early hourly mortality also did not differ.

\section{Conclusion}

Review began 03/11/2021 Review ended 03/25/2021 Published 04/13/2021

\section{() Copyright 2021}

Plurad et al. This is an open access article distributed under the terms of the Creative Commons Attribution License CC-BY 4.0., which permits unrestricted use, distribution, and reproduction in any medium, provided the original author and source are credited.
There is no overall or hourly mortality discrepancy between ACS-verified level I and II centers for patients presenting with hypotension. This potentially relates to the use of more contemporary data gathered after implementation of updated verification requirements.

Categories: Quality Improvement, Epidemiology/Public Health, Trauma

Keywords: mortality, trauma center, verification, american college of surgeons, unstable trauma

\section{Introduction}

Previous literature outlines significant outcome differences between level I and level II trauma centers. Superior outcomes are reported at level I centers for the severely injured [1,2], traumatic brain injury (TBI) patients [3,4], those with other specific injuries [5], and overall mortality [6,7]. Less prevalent are studies showing improved outcomes or equivalency at level II centers [8-11]. Of significance to our study, previous data show that trauma patients presenting with hemodynamic instability have significantly lower mortality in level I versus level II centers and that this discrepancy is sustained during the first hours of admission [2]. It was hypothesized that level II centers have access to inferior resources. However, during the time many of these investigations were being reported, there were differences in clinical requirements at level I versus level II trauma centers.

Since 1976, the American College of Surgeon Committee on Trauma (ACS-COT) has issued trauma center resource guidelines. "Resources for the Optimal Care of the Injured Patient" (resources manual) emphasizes the importance of a systems-based approach mandating escalating clinical resources at higher level trauma centers [12]. The 2014 update mandated equivalent clinical resources at level I and II centers so that, theoretically, outcomes would be similar. However, there are little recent data to support this. We hypothesize that more contemporary analysis would support improved outcomes at level II centers relative 


\section{Materials And Methods}

Utilizing the 2017 Trauma Quality Program Participant Use File (TQP-PUF), we identified adult patients (age $>14$ years) who presented to an ACS-COT verified level I or II trauma center with hemodynamic instability (systolic blood pressure [SBP] $<90 \mathrm{mmHg}$ ) [2]. We excluded patients with isolated TBI and interfacility transfers. Isolated TBI was identified by an Abbreviated Injury Scale (AIS) score for head of $\geqslant 3$ with an AIS score for all other body regions of $<3$ [3]. We extracted all pertinent demographic and injury variables. This included, but was not limited to, gender, race, E code mechanism (mechanism), admission Glasgow Coma Scale (GCS) score, Injury Severity Score (ISS), and the presence of medical co-morbidities (Charlson Comorbidity Index [CCI]). Outcome variables include ICU and hospital lengths of stay (LOS) and in-hospital mortality.

Continuous variables were converted to dichotomous variables at clinically significant cut-points. This included, but not limited to, age (> 60 years), hypoxia $\left(\mathrm{O}_{2}\right.$ saturation $<93 \%$ ), severe TBI (admission GCS $<9$ ), severe injury (ISS $>15$ ), and CCI $\geqslant 3$. Demographic and injury variables were compared between the groups admitted to a level I versus level II center. Similarly, variables were studied for their association with mortality. Univariate analysis was performed using Student's t-test or ANOVA (analysis of variance) for continuous variables and X2 for dichotomous variables. All variables with a $\mathrm{p}$-value of $<0.05$ on univariate analysis were then entered into logistic regression to determine adjusted mortality outcomes, with admission to a level II being added to the model. Results are reported as raw numbers, percentages, and odds ratios with $95 \%$ confidence intervals with p-values where appropriate. SPSS Version 21 (IBM Corp., Chicago, IL, USA) was used for statistical analysis. Comparisons were considered statistically significant with a pvalue of $<0.05$.

\section{Results}

There were 7,264 patients meeting the inclusion criteria (Figure 1). Most patients were male (4,924 [67.8\%]) and white $(4,783[65.8 \%])$. Mean age was $47.9( \pm 19.5)$ years. Primary mechanisms were occupants in motor vehicle trauma (1,808 [24.9\%]) followed by falls (1,346 [18.5\%]). The study group was severely injured with a mean ISS of $18.5( \pm 14.6)$. Mean ICU and hospital LOS were $8( \pm 9.5)$ and $11.7( \pm 15.1)$ days, respectively. There were 1,097 (15.1\%) in-hospital deaths (Table 1). 


\section{Cureus}

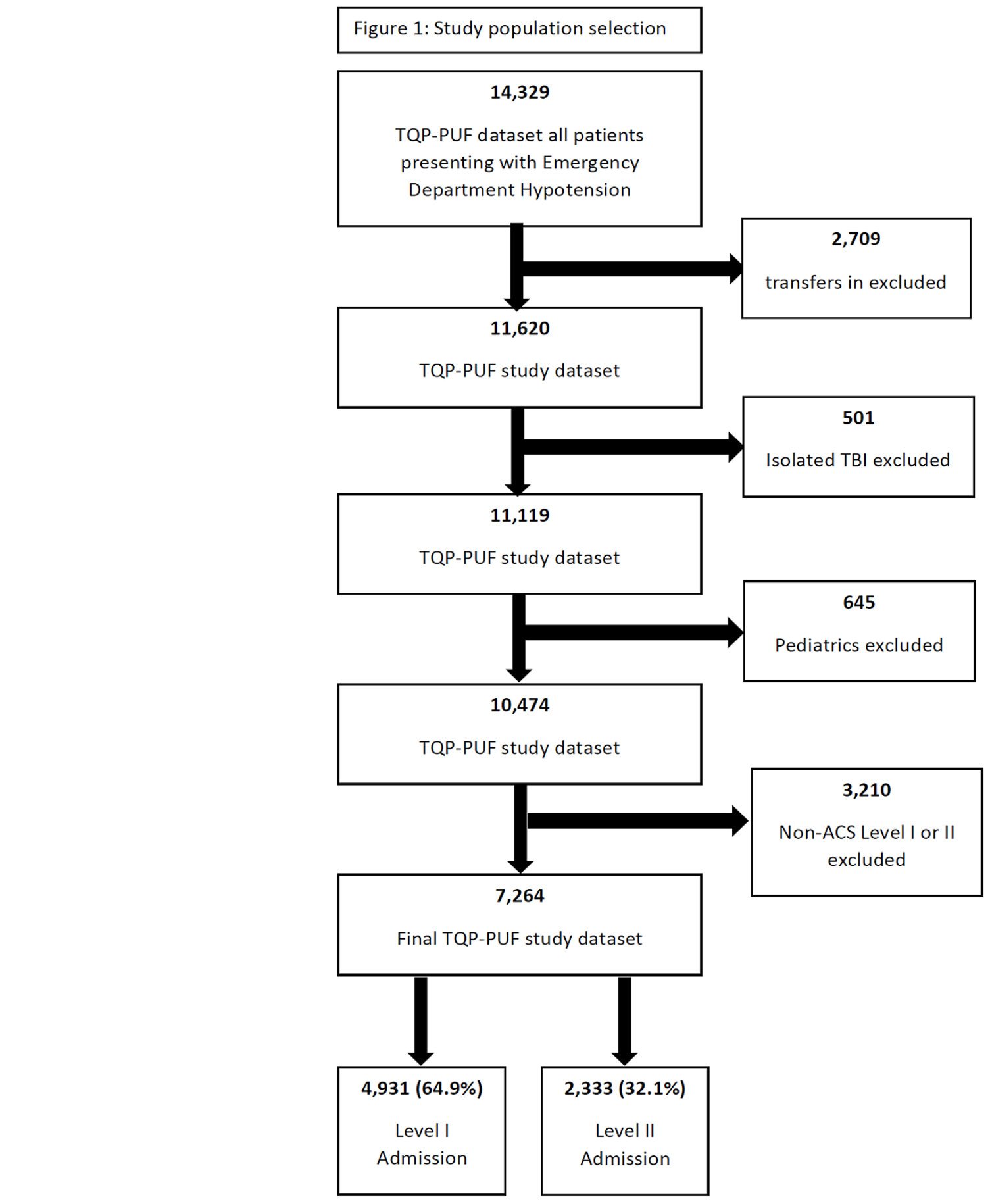

FIGURE 1: Study population selection

TQP-PUF, Trauma Quality Program Participant Use File

\section{Variable}

Study patient

Level I admission

Level II admission

Demographics

Gender (male) n or mean (\% or SD)

7,264

$4,931(67.9 \%)$

$2,333(32.1 \%)$

$4,924(67.8 \%)$ 


\section{Cureus}

Mean age (years)

Race

Asian-Pacific Islander

Black

Other

White

Mechanism (E code)

Cut/pierce

Fall

Fall from height

Firearm

MVT-occupant

MVT-motorcycle

MVT-pedestrian

Pedestrian/cyclist

Struck by/against

Other classifiable

Trauma type

Blunt

Penetrating

Thermal

Injury severity/demographics

Admission mean ISS

ISS $>15$

Mean SBP $(\mathrm{mmHg})$

Admission hypoxia $\left(\mathrm{SpO}_{2}<93 \%\right)$

TBI all

TBI mild

TBI moderate

TBI severe

Co-morbidities

Alcohol abuse disorder

Anticoagulation

CHF

Cirrhosis

COPD

CVA

Dementia

Diabetes
47.9 ( \pm 19.5$)$

147 (2.1\%)

$748(10.3 \%)$

$1,586(21.8 \%)$

$4738(65.8 \%)$

737 (10.1\%)

$1,346(18.5 \%)$

$556(7.7 \%)$

1,096 (15.1\%)

$1,808(25 \%)$

553 (7.6\%)

$478(6.7 \%)$

276 (3.9\%)

$236(3.2 \%)$

$143(2.1 \%)$

5,924 (81.6\%)

1,318 (18.1\%)

$22(0.3 \%)$

$18.5( \pm 14.6)$

3,652 (50.3\%)

$73.2( \pm 13)$

$2,032(28 \%)$

909 (12.5\%)

$130(1.8 \%)$

$993(13.7 \%)$

757 (10.4\%)

$462(6.4 \%)$

276 (3.8\%)

159 (2.2\%)

467 (6.4\%)

142 (2\%)

$122(1.7 \%)$

$846(11.6 \%)$ 


\section{Cureus}

HTN

$1,872(25.8 \%)$

Other

$1,068(14.7 \%)$

Psychiatric disorder

939 (12.9\%)

Renal dysfunction

$131(1.8 \%)$

Substance abuse

$783(10.8 \%)$

Chronic condition (any)

$5,111(70.4 \%)$

Mean CCI

$1.54( \pm 2.1)$

Trauma center characteristics

University teaching

4,066 (56\%)

Community teaching

$2,472(34 \%)$

Non-teaching

$726(10 \%)$

$<200$ beds

419 (5.8\%)

201-400 beds

$2,013(27.7 \%)$

401-600 beds

$1,978(27.2 \%)$

$>600$ beds

$2,854(39.3 \%)$

Primary payor characteristics

Medicare/Medicaid

$3,189(43.9 \%)$

Private

$2,566(35.3 \%)$

Other

$344(4.7 \%)$

Uninsured

$1,165(16.1 \%)$

Treatment after ED

Ward

$1,993(27.4 \%)$

OR

$2,395(33 \%)$

ICU

$2,722(37.5 \%)$

Death in ED

$154(2.1 \%)$

Outcome

ICU LOS (days)

$8( \pm 9.8)$

Hospital LOS (days)

$11.7( \pm 13.9)$

Deaths

$1,097(15.1 \%)$

TABLE 1: Hypotensive (SBP $<90 \mathrm{mmHg}$ ) patients admitted to an ACS-COT level I or II trauma center

Data are expressed as raw numbers, percentages, and means with standard deviations.

ACS-COT, American College of Surgeon Committee on Trauma; CCI, Charlson Comorbidity Index; CHF, congestive heart failure; COPD, chronic obstructive pulmonary disease; CVA, cerebrovascular accident; ED, emergency department; HTN, hypertension; ICU, intensive care unit; ISS, Injury Severity Score; LOS, length of stay; MVT, motor vehicle traffic; OR, operating room; SBP, systolic blood pressure; TBI, traumatic brain injury 


\section{Cureus}

surgery or angiography for hemorrhage control (not shown in table) (34.5\% vs. $25.3 \%$; $\mathrm{p}<0.001)$. The presence of a TBI was similar (27.8\% vs. $28.3 \%$; $\mathrm{p}=0.980$ ). ICU LOS was not different but hospital LOS was longer at a level I center (mean $12.1\left[{ }^{ \pm 14}\right]$ vs. $10.9[ \pm 13.6]$ days; $\left.\mathrm{p}<0.001\right)$. Mortality was higher at a Level I center; however, this was not statistically significant $(15.5 \%$ vs. $14.2 \%$, $\mathrm{p}=0.134)$.

\begin{tabular}{|c|c|c|c|}
\hline Variable & Level I: 4,931 (67.9\%) & Level II: 2,333 (32.1\%) & OR/mean difference $(95 \% \mathrm{Cl})$, p-value \\
\hline \multicolumn{4}{|l|}{ Demographics } \\
\hline Gender (male) & $3,389(68.7 \%)$ & $1,535(65.8 \%)$ & 1.143 (1.029- 1.269), 0.012 \\
\hline Mean age (years) & $46.8( \pm 19)$ & $50.3( \pm 20.1)$ & $-3.467(-4.423$ to 2.511$),<0.001$ \\
\hline \multicolumn{4}{|l|}{ Race } \\
\hline Asian-Pacific Islander & $111(2.3 \%)$ & $36(1.5 \%)$ & $<0.001$ \\
\hline Black & $476(9.7 \%)$ & $272(11.7 \%)$ & \\
\hline Other & $1,225(24.8 \%)$ & $361(15.5 \%)$ & \\
\hline White & $3,119(63.3 \%)$ & $1,664(71.3 \%)$ & \\
\hline \multicolumn{4}{|l|}{ Mechanism (E code) } \\
\hline Cut/pierce & $525(10.6 \%)$ & $212(9.1 \%)$ & $<0.001$ \\
\hline Fall & $781(15.8 \%)$ & $565(24.2 \%)$ & \\
\hline Fall from height & $360(7.3 \%)$ & $196(8.4 \%)$ & \\
\hline Firearm & $833(16.9 \%)$ & $263(11.3 \%)$ & \\
\hline MVT occupant & $1,362(25.6 \%)$ & $546(23.4 \%)$ & \\
\hline MVT motorcycle & $280(7.7 \%)$ & $173(7.4 \%)$ & \\
\hline MVT pedestrian & $327(6.6 \%)$ & $151(6.5 \%)$ & \\
\hline Pedestrian/cyclist & $187(3.8 \%)$ & $89(3.8 \%)$ & \\
\hline Struck by/against & $151(3.1 \%)$ & $85(3.6 \%)$ & \\
\hline Other classifiable & $101(2 \%)$ & $42(1.8 \%)$ & \\
\hline \multicolumn{4}{|l|}{ Trauma type } \\
\hline Blunt & $3,980(80.7 \%)$ & $1,944(83.3 \%)$ & 0.015 \\
\hline Penetrating & $933(18.9 \%)$ & $385(16.5 \%)$ & \\
\hline Thermal & $18(0.4 \%)$ & $4(0.2 \%)$ & \\
\hline \multicolumn{4}{|l|}{ Injury severity } \\
\hline Mean ISS & $19.3( \pm 15)$ & $16.7( \pm 13.7)$ & $2.611(1.893-3.329),<0.001$ \\
\hline ISS > 15 & $2,614(53 \%)$ & $1,038(44.5 \%)$ & $1.408(1.275-1.554),<0.001$ \\
\hline Admission mean SBP (mmHg) & $73.4( \pm 18.5)$ & $72.8( \pm 20)$ & $0.637(-0.300$ to 1.574$), 0.183$ \\
\hline Admission hypoxia $\left(\mathrm{SpO}_{2}<93 \%\right)$ & $857(42.8 \%)$ & $420(18 \%)$ & 0.961 (0.845-1.093), 0.543 \\
\hline TBl all & $1,372(27.8 \%)$ & $660(28.3 \%)$ & $0.977(0.876-1.090), 0.980$ \\
\hline TBI mild & $587(11.9 \%)$ & $322(13.8)$ & $<0.001$ \\
\hline TBI moderate & $96(1.9 \%)$ & $34(1.5 \%)$ & \\
\hline TBI severe & $689(14 \%)$ & $304(13 \%)$ & \\
\hline \multicolumn{4}{|l|}{ Co-morbidities } \\
\hline Alcohol abuse disorder & $515(10.4 \%)$ & $242(10.4 \%)$ & $1.008(0.858-1.184), 0.929$ \\
\hline Anticoagulation & $294(6 \%)$ & $168(7.2 \%)$ & $0.817(0.671-0.994), 0.043$ \\
\hline
\end{tabular}




\section{Cureus}

\begin{tabular}{|c|c|c|c|}
\hline $\mathrm{CHF}$ & $180(3.7 \%)$ & $96(4.1 \%)$ & 0.883 (0.686-1.137), 0.334 \\
\hline Cirrhosis & $116(2.4 \%)$ & $43(1.8 \%)$ & 1.283 (0.901-1.827), 0.166 \\
\hline COPD & $306(6.2 \%)$ & $161(6.9 \%)$ & 0.893 (0.733-1.087), 0.259 \\
\hline CVA & $85(1.7 \%)$ & $57(2.4 \%)$ & 0.700 (0.499-0.983), 0.039 \\
\hline Dementia & $74(1.5 \%)$ & $48(2.1 \%)$ & 0.725 (0.503-1.046), 0.085 \\
\hline Diabetes & $523(10.6 \%)$ & $323(13.8 \%)$ & $0.738(0.637-0.856),<0.001$ \\
\hline HTN & $1,171(23.7 \%)$ & $701(30 \%)$ & $0.725(0.649-0.809),<0.001$ \\
\hline Other & $700(14.2 \%)$ & $368(15.8 \%)$ & 0.770 (1.013-1.013), 0.076 \\
\hline Psychiatric disorder & $652(13.2 \%)$ & $287(12.3 \%)$ & 1.086 (0.936-1.260), 0.275 \\
\hline Renal dysfunction & $89(1.8 \%)$ & $42(1.8 \%)$ & 1.003 (0.692-1.452), 0.989 \\
\hline Substance abuse & $561(11.4 \%)$ & $222(9.5 \%)$ & 1.221 (1.036-1.438), 0.017 \\
\hline Chronic condition (any) & $3,418(69.3 \%)$ & $1,693(72.6 \%)$ & 0.854 (0.766-0.953), 0.005 \\
\hline Mean CCl & $1.43( \pm 2)$ & $1.77( \pm 2.2)$ & $0.695(0.623-0.773),<0.001$ \\
\hline \multicolumn{4}{|c|}{ Trauma center characteristics } \\
\hline University teaching & $3,802(77.1 \%)$ & $279(12 \%)$ & $<0.001$ \\
\hline Community teaching & $1,139(22.9 \%)$ & $1,343(57.3 \%)$ & \\
\hline Non-teaching & $0(0 \%)$ & $711(30.5 \%)$ & \\
\hline$<200$ beds & $202(4.1 \%)$ & $217(9.3 \%)$ & $<0.001$ \\
\hline $201-400$ beds & $631(12.8 \%)$ & $1,382(59.2 \%)$ & \\
\hline $401-600$ beds & $1,625(33 \%)$ & $353(15.1 \%)$ & \\
\hline$>600$ beds & $2,473(50.2 \%)$ & $381(16.3 \%)$ & \\
\hline \multicolumn{4}{|c|}{ Primary payor characteristics } \\
\hline Medicare/Medicaid & $2,148(43.6 \%)$ & $1,041(44.6 \%)$ & $<0.001$ \\
\hline Private insurance & $1,651(33.5 \%)$ & $915(39.2 \%)$ & \\
\hline Uninsured & $901(18.3 \%)$ & $264(11.3 \%)$ & \\
\hline Other & $231(4.7 \%)$ & $113(4.8 \%)$ & \\
\hline \multicolumn{4}{|l|}{ Treatment after ED } \\
\hline Ward & $1,274(25.8 \%)$ & $719(30.8 \%)$ & $<0.001$ \\
\hline OR & $1,700(34.5 \%)$ & $695(29.8 \%)$ & \\
\hline ICU & $1,842(37.4 \%)$ & $880(37.7 \%)$ & \\
\hline Death in ED & $115(2.3 \%)$ & $39(1.7 \%)$ & 1.405 (0.974-2.026), 0.081 \\
\hline \multicolumn{4}{|l|}{ Outcomes } \\
\hline ICU LOS (days) & $7.9( \pm 9.2)$ & $8.2( \pm 10.2)$ & $-0.262(-0.843$ to 0.318$), 0.376$ \\
\hline Hospital LOS (days) & $12.1( \pm 14)$ & $10.9( \pm 13.6)$ & $1.269(0.587-1.951),<0.001$ \\
\hline Mortality & 766 (15.5\%) & $331(14.2 \%)$ & $1.112(0.968-1.279), 0.134$ \\
\hline
\end{tabular}

TABLE 2: Comparison of admissions to level I versus level II trauma centers for hypotensive (SBP $<90 \mathrm{mmHg}$ ) patients

Data are expressed as raw numbers, percentages, and means with standard deviations.

$\mathrm{CCI}$, Charlson Comorbidity Index; CHF, congestive heart failure; COPD, chronic obstructive pulmonary disease; CVA, cerebrovascular accident; ED, 


\section{Cureus}

emergency department; HTN, hypertension; ICU, intensive care unit; ISS, Injury Severity Score; LOS, length of stay; MVT, motor vehicle traffic; OR, operating room; SBP, systolic blood pressure; TBI, traumatic brain injury

Deaths are compared to survivors in Table 3. Males $(73.2 \%$ vs. $66.8 \%$; $\mathrm{p}<0.001)$ and victims of firearm injuries $(20.4 \%$ vs. $14.1 \%,<0.001)$ were more likely to die. The presence of a TBI $(57.1 \%$ vs. $23 \%,<0.001)$ and higher mean ISS (32.6 [ \pm 17.1$]$ vs. $16[ \pm 12.6], p<0.001)$ were also associated with increased mortality. Increasing instability, reflected in lower mean admission SBP, also predicted death (mean SBP: 60.3 [ \pm 29.6$]$ vs. 75.5 $[ \pm 15.4] \mathrm{mmHg} ; \mathrm{p}<0.001)$. Co-morbidities (CCI: $1.64( \pm 2.28)$ vs. 1.52 [2.02]; $\mathrm{p}=0.081)$ ) were not statistically associated with death on univariate analysis, though age $>60$ years $(34.1 \%$ vs. $29.7 \%$; $\mathrm{p}=0.003)$ predicted mortality.

\section{Variable}

Demographics

Gender (male)

Mean age (years)

Age $>60$ years

Race

Asian-Pacific Islander

Black

Other

White

Mechanism (E code)

Cut/pierce

Fall

Fall from height

Firearm

MVT occupant

MVT motorcycle

MVT pedestrian

Pedestrian/cyclist

Struck by/against

Other classifiable

Trauma type

Blunt

Penetrating

Thermal

Injury severity

Mean ISS

ISS $>15$

Admission mean SBP $(\mathrm{mmHg})$

Admission Hypoxia $\left(\mathrm{SpO}_{2}<93 \%\right)$

TBI all

TBI mild
Survivor: 6,167 (84.9\%)

$4,121(66.8 \%)$

$47( \pm 19.1)$

$1,830(29.7 \%)$

$123(2 \%)$

$640(10.4 \%)$

$1,348(21.9 \%)$

$4,056(65.8 \%)$

$693(11.2 \%)$

$101(9.2 \%)$

$1,245(20.2 \%)$

$84(7.7 \%)$

$472(7.7 \%)$

$224(20.4 \%)$

$872(14.1 \%)$

$316(28.8 \%)$

$1,492(24.2 \%)$

$110(10 \%)$

$443(7.2 \%)$

105 (9.6\%)

$373(6 \%)$

$50(4.6 \%)$

$226(3.7 \%)$

22 (2\%)

214 (3.4\%)

$21(2.8 \%)$

$111(1.8 \%)$

$903(82.3 \%)$

$5,021(81.4 \%)$

$1,130(18.3 \%)$

$16(0.3 \%)$

$6(0.5 \%)$

$32.6( \pm 17.1)$

$16( \pm 12.6)$

$960(87.5 \%$

$2,692(43.7 \%)$

75.5 ( \pm 15.4$)$

904 (14.7\%)

$1,416(23 \%)$

839 (13.6\%)
0.924

$<0.001$

0.480

1.356 (1.174-1.566), $<0.001$

$0.216(-1.035$ to 1.466$), 0.735$

1.226 (1.070-1.405), 0.003

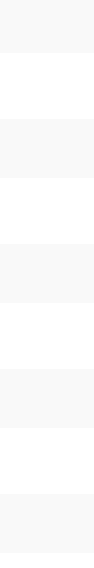

16.606 (15.748-17.465), $<0.001$

9.045 (7.511-10.894), <0.001

$-15.253(-16.424$ to 14.083$),<0.001$

3.024 (2.620-3.490), <0.001

4.297 (3.761-4.909), $<0.001$

$<0.001$ 


\section{Cureus}

\begin{tabular}{|c|c|c|c|}
\hline TBI moderate & $11(1 \%)$ & 119 (1.9\%) & \\
\hline TBI severe & $532(48.5 \%)$ & 458 (7.4\%) & \\
\hline \multicolumn{4}{|l|}{ Co-morbidities } \\
\hline Alcohol abuse disorder & $64(5.8 \%)$ & $693(11.2 \%)$ & $0.489(0.376-0.638),<0.001$ \\
\hline Anticoagulation & $57(5.2 \%)$ & $405(6.6 \%)$ & 0.780 (0.586-1.037), 0.086 \\
\hline CHF & 39 (3.6\%) & 237 (3.8\%) & 0.922 (0.653-1.302), 0.646 \\
\hline Cirrhosis & $43(3.9 \%)$ & $116(1.9 \%)$ & 2.128 (1.491-3.038), <0.001 \\
\hline COPD & 47 (4.3\%) & $420(6.8 \%)$ & 0.612 (0.450-0.834), 0.002 \\
\hline CVA & $11(1 \%)$ & $131(2.1 \%)$ & 0.467 (0.251-0.866), 0.013 \\
\hline Dementia & $18(1.6 \%)$ & $104(1.7 \%)$ & 0.973 (0.587-1.611), 0.914 \\
\hline Diabetes & $89(8.1 \%)$ & $757(12.3 \%)$ & $0.631(0.502-0.794),<0.001$ \\
\hline HTN & $193(17.6 \%)$ & $1,679(27.2 \%)$ & 0.571 (0.484-0.673), 0.001 \\
\hline Other & $153(13.9 \%)$ & $915(14.8 \%)$ & 0.930 (0.773-1.119), 0.443 \\
\hline Psychiatric disorder & $85(7.7 \%)$ & $854(13.8 \%)$ & $0.523(0.414-0.660),<0.001$ \\
\hline Renal dysfunction & $26(2.4 \%)$ & $105(1.7 \%)$ & 1.402 (0.908-2.164), 0.126 \\
\hline Substance abuse & $59(5.4 \%)$ & $724(11.7 \%)$ & $0.427(0.325-0.532),<0.001$ \\
\hline Chronic condition (any) & $670(61.1 \%)$ & 4,441 (72\%) & $0.610(0.534-0.697),<0.001$ \\
\hline Mean CCl & $1.64( \pm 2.28)$ & $1.52( \pm 2.02)$ & $0.118(-0.014$ to 0.250$), 0.081$ \\
\hline \multicolumn{4}{|c|}{ Trauma center characteristics } \\
\hline Level I trauma center & $766(69.8 \%)$ & $4,165(67.5 \%)$ & 1.112 (0.968-1.279), 0.141 \\
\hline University teaching & $657(59.9 \%)$ & $3,424(55.5 \%)$ & $<0.001$ \\
\hline Community teaching & $343(31.3 \%)$ & $2,129(34.6 \%)$ & \\
\hline Non-teaching & 97 (8.8\%) & $614(10 \%)$ & \\
\hline$<200$ beds & $66(6 \%)$ & $353(5.7 \%)$ & $<0.001$ \\
\hline $201-400$ beds & $282(25.7 \%)$ & $1,731(28.1 \%)$ & \\
\hline $401-600$ beds & $291(26.5 \%)$ & $1,687(27.4 \%)$ & \\
\hline$>600$ beds & $458(41.8 \%)$ & $2,396(38.9 \%)$ & \\
\hline \multicolumn{4}{|c|}{ Primary payor characteristics } \\
\hline Medicare/Medicaid & 396 (36.1\%) & $2,793(45.3 \%)$ & $<0.001$ \\
\hline Private insurance & 362 (33\%) & 2,204 (35.7\%) & \\
\hline Uninsured & $280(25.5 \%)$ & 885 (14.4\%) & \\
\hline Other & $59(5.4 \%)$ & $285(4.6 \%)$ & \\
\hline
\end{tabular}

\section{TABLE 3: Comparison of deaths and survivors for hypotensive (SBP $<90 \mathrm{mmHg}$ ) trauma patients}

Data are expressed as raw numbers, percentages, and means with standard deviations.

CCI, Charlson Comorbidity Index; CHF, congestive heart failure; COPD, chronic obstructive pulmonary disease; CVA, cerebrovascular accident; HTN, hypertension; ISS, Injury Severity Score; MVT, motor vehicle traffic; SBP, systolic blood pressure; TBI, traumatic brain injury 


\section{Cureus}

to a level I versus a level II center was not (1.009 [0.851-1.196]; $p=0.918)$. The hourly risk of death, similarly, was not statistically significant with admission to a level I versus II center (Figure 2).

\section{Variable}

Level I versus level II

GCS $<9$

ISS $>15$

Age $>60$ years

Hypoxia $\left(\mathrm{SpO}_{2}<93 \%\right)$

Primary payor

Mechanism (E Code)

TBI any

\section{$\operatorname{Exp}(B)(95 \% \mathrm{Cl}$ for $\operatorname{Exp}(B)), p$-value}

1.009 (0.851-1.196), 0.918

$10.806(9.019-12.947),<0.001$

$4.508(3.638-5.590),<0.001$

3.821 (3.142-4.646), <0.001

1.901 (1.596-2.264), <0.001

$0.799(0.729-0.876),<0.001$

1.44 (1.167-1.778), 0.001

$1.323(1.110-1.577), 0.002$

\section{TABLE 4: Adjusted mortality outcomes for hypotensive trauma patients}

Other variables entered into forward stepwise regression: gender, race, $\mathrm{CCl}$, hospital teaching type, hospital size

GCS Glasgow Coma Scale; ISS, Injury Severity Score; TBI, traumatic brain injury

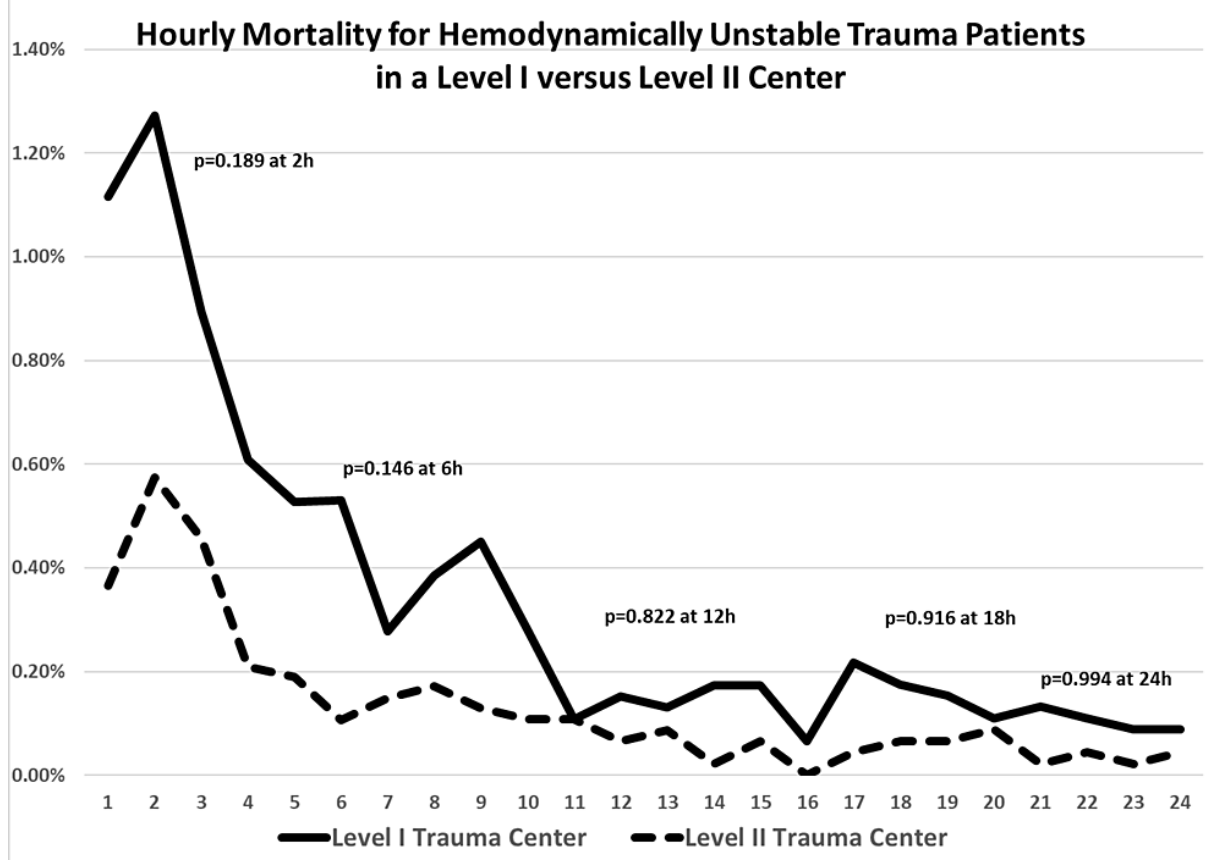

FIGURE 2: Hourly mortality for hemodynamically unstable trauma patients in a level I versus level II center

\section{Discussion}

Literature is replete with studies addressing the outcome differences between level I and II trauma centers. Significant mortality increases at level II centers for specific subsets have been demonstrated. These include the severely injured [1,2], those with specific wounding [5], those with TBI [8,11], those transferred to a level II center after TBI [13], and overall populations [6,7]. Of specific interest to our study, those admitted with hemodynamic instability have been shown to have better mortality outcomes at a level I center that is sustained through early admission [2]. Of concern is that these reports may utilize obsolete data gathered prior to the 2014 "resources manual" update [12]. The previously mentioned study [2] on hypotensive 
trauma patients is an example of one of the more egregious confounding as it utilized data nearly over a decade old to support conclusions that were no longer valid at the time of publication.

The 2014 revision of the ACS-COT resources manual (Orange Book) specifically addresses clinical resourcing discrepancies between level I and II ACS-COT verified centers. "Level I and II criteria were revised to ensure that level I and II trauma centers are available to provide high quality definitive care" [12]. The assumption is that this would lead to reduction or elimination outcome discrepancies, though there are little data to support this. Our current investigation demonstrates this relative improvement and contradicts earlier reports. Furthermore, previous studies that demonstrate outcome equivalence or even improved outcomes at level II centers used locoregional data [8,14].

Our investigation shows no mortality difference between admission to a level I versus level II center in patients who present with hemodynamic instability. We hypothesize that this is associated with utilization of more contemporary datasets that were gathered after implementation of the 2014 ACS-COT "resources manual". Updated requirements include, but are not limited to, uninterrupted emergency medicine staffing, more stringent operating room resourcing with performance tracking, defined minimums for highest level activations, minimum registrar training requirements, dedicated injury prevention positions, equivalent surgical and non-surgical subspecialty services, and changes in guidance for consultant bedside presence. One of the more pertinent improvements for both level I and II centers relate to performance improvement mandating exacting identification and trending of important outcome and process metrics. In addition, participation in a risk-adjusted outcome benchmarking program (Trauma Quality Improvement Program [TQIP]) was a significant new mandate [15]. Elements for a level I center, not required of a level II center, are admission volume minimums, the presence of higher level surgical resident trainees, a surgically directed intensive care unit, and minimum research productivity.

Despite a reasonably valid clinical association between improved resources and better outcomes, our study is hindered by its retrospective design. Trauma care at level II centers could have simply improved over time with the introduction of new techniques and protocols, though this is doubtful relative to outcome improvements related to mandated improved resourcing. While the 2017 TQP-PUF is a powerful tool to assess the impact of sweeping administrative mandates, it lacks the granularity to define elements that may have had an impact on our findings or that could have contributed to confounding. There were significant differences between level I and level II admissions. Most notably, level I admissions were more severely injured and differed demographically and by mechanism. Despite significant differences, adjusted mortality that included these variables was similar between level I and II centers and sustained hourly through the first 24 hours of admission. Of note, co-morbidities were not associated with death. This could possibly be due to survivors having more opportunity for their care teams to identify and document co-morbidities.

Applicability of our study extends only to ACS-COT verified centers regardless of the designating authority. While the scope of this organization is broad and many local designating authorities model their verification efforts to mirror the ACS-COT process and requirements, not all designated trauma centers are verified by this committee. It may be reasonable to assume that level II trauma centers verified by their local designating authority or other non-ACS-COT construct also experience improved outcomes relative to level I; however, our study excluded these trauma centers.

Despite these shortcomings, our study is impactful since it demonstrates, in contradiction to older published data, that level II trauma centers achieve similar outcomes in patients who present with hemodynamic instability compared to level I centers. In this subgroup, immediate presence of trauma surgeons, competent consultants, and timely availability of interventions are critical [15]. This change likely relates, in part, to the updated requirements that these elements be in place at a level II center just as they are at the level I center. Additionally, our study demonstrates the potentially significant and widespread beneficial impact of ongoing process improvement, resource standardization, and involvement of a national verification program. It is important for the public and policy-makers since it supports that the significant investment in a level II can be expected to generate outcomes similar to those at a level I center.

\section{Conclusions}

As opposed to previous studies, level II trauma centers perform similar to level I centers for trauma patients who present with hemodynamic instability. This may relate to compliance with the ACS-COT resources manual, Resources for the Optimal Care of the Injured Patient, 2014 version. Further study would include examination of trauma centers that are not verified by the ACS-COT that could reveal more specific variables associated with improved outcome in these patients.

\section{Additional Information \\ Disclosures}

Human subjects: All authors have confirmed that this study did not involve human participants or tissue. Animal subjects: All authors have confirmed that this study did not involve animal subjects or tissue. Conflicts of interest: In compliance with the ICMJE uniform disclosure form, all authors declare the 
following: Payment/services info: All authors have declared that no financial support was received from any organization for the submitted work. Financial relationships: All authors have declared that they have no financial relationships at present or within the previous three years with any organizations that might have an interest in the submitted work. Other relationships: All authors have declared that there are no other relationships or activities that could appear to have influenced the submitted work.

\section{References}

1. Glance LG, Osler TM, Mukamel DB, Dick AW: Impact of trauma center designation on outcomes: is there a difference between level I and level II trauma centers?. J Am Coll Surg. 2012, 215:372-8. 10.1016/j.jamcollsurg.2012.03.018

2. Herrera-Escobar JP, Rios-Diaz AJ, Zogg CK, et al.: The "mortality ascent": hourly risk of death for hemodynamically unstable trauma patients at level II versus level I trauma centers. J Trauma Acute Care Surg. 2018, 84:139-145. 10.1097/ta.0000000000001706

3. DuBose IJ, Browder T, Inaba K, Teixeira PG, Chan LS, Demetriades D: Effect of trauma center designation on outcome in patients with severe traumatic brain injury. Arch Surg. 2008, 143:1213-7. 10.1001/archsurg.143.12.1213

4. Chalouhi N, Mouchtouris N, Saiegh FA, Starke RM, Theofanis T, Das SO, Jallo J: Comparison of outcomes in level I vs level II trauma centers in patients undergoing craniotomy or craniectomy for severe traumatic brain injury. Neurosurgery. 2020, 86:107-11. 10.1093/neuros/nyy634

5. Demetriades D, Martin M, Salim A, Rhee P, Brown C, Chan L: The effect of trauma center designation and trauma volume on outcome in specific severe injuries. Ann Surg. 2005, 242:512-7. 10.1097/01.sla.0000184169.73614.09

6. Cudnik MT, Newgard CD, Sayre MR, Steinberg SM: Level I versus level II trauma centers: an outcomesbased assessment. J Trauma. 2009, 66:1321-6. 10.1097/ta.0b013e3181929e2b

7. Brown JB, Watson GA, Forsythe RM, et al.: American College of Surgeons trauma center verification versus state designation: are level II centers slipping through the cracks? J Trauma Acute Care Surg. 2013, 75:44-9. 10.1097/TA.0b013e3182988729

8. Kaji AH, Bosson N, Gausche-Hill M, Dawes AJ, Putnam B, Shepherd T, Lewis RJ: Patient outcomes at urban and suburban level I versus level II trauma centers. Ann Emerg Med. 2017, 70:161-8. 10.1016/j.annemergmed.2017.01.040

9. Elkbuli A, Dowd B, Flores R, Boneva D, McKenney M: The impact of level of the American College of Surgeons Committee on Trauma verification and state designation status on trauma center outcomes. Medicine (Baltimore). 2019, 98:16133. 10.1097/MD.0000000000016133

10. Ehrlich PF, Rockwell S, Kincaid S, Mucha P Jr: American College of Surgeons, Committee on Trauma Verification Review: does it really make a difference?. J Trauma. 2002, 53:811-6. 10.1097/00005373200211000-00001

11. Rogers FB, Osler T, Lee JC, et al.: In a mature trauma system, there is no difference in outcome (survival) between level I and level II trauma centers. I Trauma. 2011, 70:1354-7. 10.1097/ta.0b013e3182183789

12. Resources for Optimal Care of the Injured Patient 2014 (6th edition) Verification change log . (2020). Accessed: January 17, 2020: http://facs.org/quality=programs/trauma/.tqp/center-programs/vrc/resources.

13. McConnell KJ, Newgard CD, Mullins RJ, Arthur M, Hedges JR: Mortality benefit of transfer to level I versus level II trauma centers for head-injured patients. Health Serv Res. 2005, 40:435-57.

14. Matsushima K, Schaefer EW, Won EJ, Armen SB: The outcome of trauma patients with do-not-resuscitate orders. J Surg Res. 2016, 200:631-6. 10.1016/j.jss.2015.09.024

15. The Trauma Quality Improvement Program . (2020). Accessed: May 15, 2020: https://www.facs.org/qualityprograms/trauma/tqp/center-programs/tqip. 